\title{
Duplication of the Posterior Cerebral Artery and the 'True Fetal' Variant
}

\author{
Hesham Masoud ${ }^{a, c}$ Thanh N. Nguyen ${ }^{a-c}$ Joshua Thatcher ${ }^{c}$ \\ Glenn Barest ${ }^{c} \quad$ Alexander M. Norbash ${ }^{c}$ \\ Departments of a Neurology, ${ }^{a}$ Neurosurgery, and ${ }^{\mathrm{C}}$ Radiology, Boston Medical Center, \\ Boston, Mass., USA
}

\section{Key Words}

Stroke · Vascular embryology · Anatomy · Posterior cerebral artery · Fetal posterior cerebral artery - Duplicate posterior cerebral artery - Circle of Willis - Magnetic resonance angiography $\cdot 3 \mathrm{D}$ computed tomography angiography

\begin{abstract}
We present a rare case of bilateral posterior cerebral artery variant anatomy seen in a patient presenting with acute ischemic stroke. An embryological explanation of the variant configuration is discussed along with demonstrative radiologic images and a display of the vascular territory supplied.

(C) 2015 S. Karger AG, Basel
\end{abstract}

\section{Introduction}

The duplication of the posterior cerebral artery (PCA) is an exceedingly rare anatomical variant. Review of the literature yielded only 1 report with digitally subtracted angiographic exhibition of the variant anatomy [1]. Here, we present a case demonstrating the appearance of a duplicated PCA and provide an explanation of its true representation. In addition, a brief overview of cranial posterior circulation embryology is provided.

\section{Case Report}

A 77-year-old male presented with sudden-onset, right-upper-extremity weakness. Magnetic resonance imaging disclosed multiple foci of diffusion restriction within the left frontal and parietal lobes and parietooccipital region. Computed tomography angiography (CTA) revealed variant circle of Willis and PCA anatomy 
Fig. 1. 3D volume-rendered reconstruction of the CTA showing the usual right 'fetal' PCA (arrow) and a prominent thalamo-perforator (arrowhead). The left anterior choroidal artery (double arrows) gives rise to the parietooccipital branches, while the ipsilateral PCA supplies the calcarine branches, representing the 'true fetal PCA'.

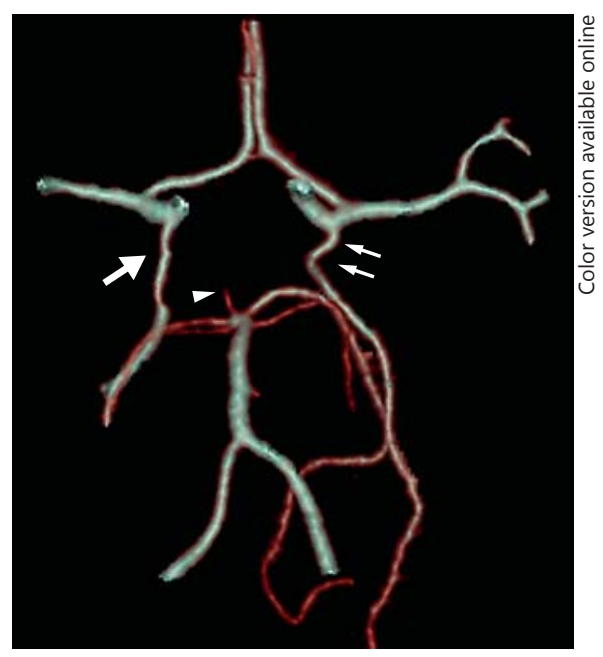

(fig. 1) as well as severe left extracranial internal carotid artery (ICA) stenosis (not shown). This stenosis was deemed to be the embolic source, and a carotid endarterectomy was performed. The patient was seen at follow-up, and no residual neurologic deficit or recurrent event was reported.

\section{Discussion}

The PCA stem is formed of the posterior choroidal branches feeding the dien-mesencephalon and it acquires its telencephalic branches by annexing the distal anterior choroidal supply [2]. The proximal PCA connection to the basilar artery usually occurs after the paired longitudinal neural arteries fuse, and the developing vertebrobasilar system begins its contribution to the PCA supply. The balance between the early anterior and posterior choroidal branches is a determinant of the degree of observed variance from the usual adult cerebrovascular configuration.

The most commonly encountered PCA variant is the so-called 'fetal PCA', where by the P2 segment of the PCA appears to be a continuation of the posterior communicating artery (Pcomm). Often this is seen with a hypoplastic or absent ipsilateral P1 PCA segment. This 'fetal configuration' has been reported to be present in up to $22 \%$ of the cases [7]. Embryologically, this can be explained by the derivation of the posterior cerebral vascular territory from the caudal trunk of the primitive ICA. Failure of the Pcomm (a remnant of the caudal embryonic ICA) to regress during this stage results in hypoplasia or agenesis of the P1 PCA segment that serves to connect the basilar artery to the PCA. Consequentially, the flow into the PCA becomes reliant on the ICA.

In fact, this variant does not truly represent a fetal origin of the PCA and has been better characterized as a persistent carotid-basilar anastomosis [5]. A true 'fetal PCA' is encountered when the fetal supply of the telencephalon via the anterior choroidal artery persists into adulthood rather than being annexed by the posterior choroidal artery. This appearance can give the false impression of a duplicated PCA [9].

Our case illustrates both representations of 'fetal' PCAs in the same patient seen on 3D volume-rendered reconstructed CTA. On the right, a dominant Pcomm is seen to continue as ipsilateral PCA branches with an absent P1 segment (fig. 1). On the left, the anterior choroidal artery gives rise to the parieto-occipital branches (fig. 2a), and the ipsilateral PCA originating from the basilar trunk gives rise to the medial calcarine artery (fig. $2 \mathrm{~b}$ ). 

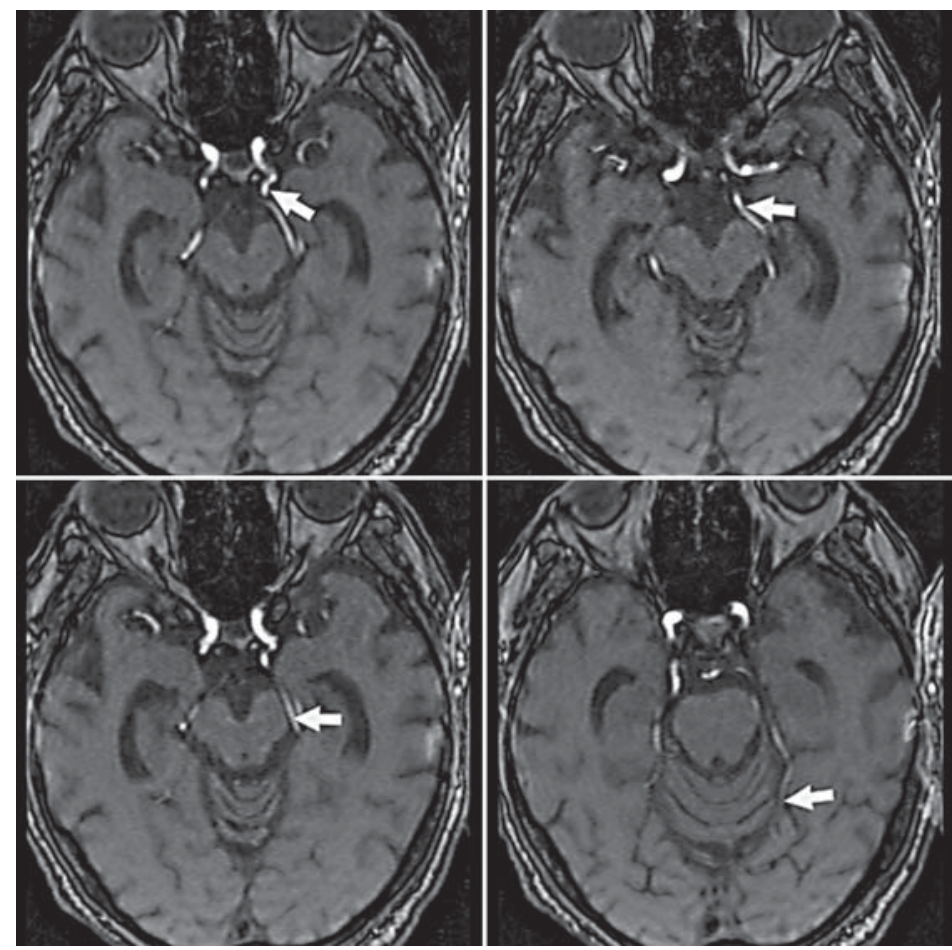

b
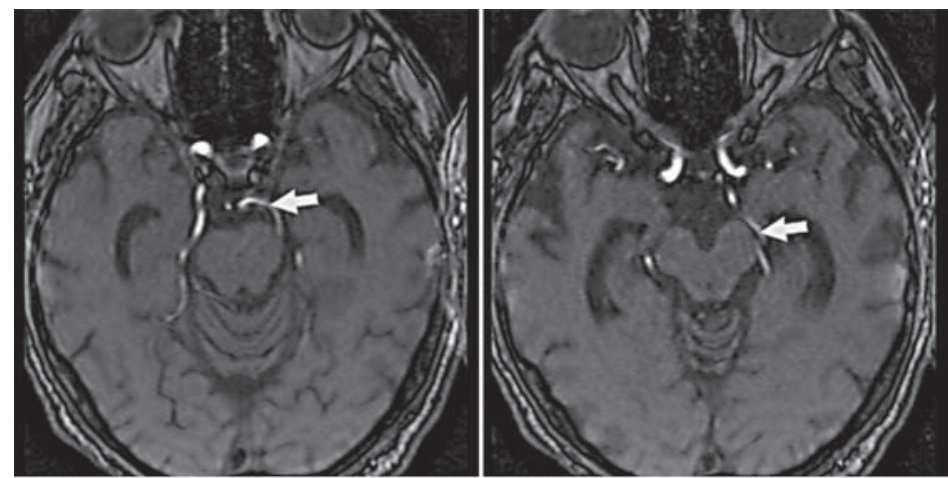

Fig. 2. Axial MRA showing the left anterior choroidal artery supplying the parieto-occipital region (a; arrow) and the left PCA, giving rise to the calcarine artery $(\mathbf{b}$; arrow).
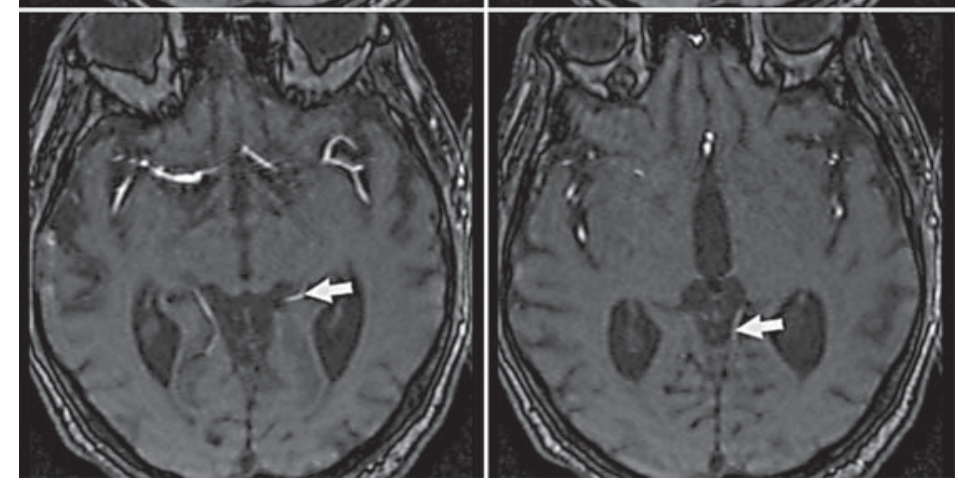

\section{Conclusion}

To our knowledge, this is the only reported case where both PCA anatomical variants are present in the same patient. The knowledge of these possible variations in adult anatomy is critical to neurointerventionists and vascular neurologists alike; the awareness of the possi- 
bility of this variant is especially relevant in cases of embolic stroke where posterior circulation territorial infarcts can be attributed to an anterior circulation source (e.g., symptomatic extracranial carotid stenosis) rather than cardioembolism.

\section{Disclosure Statement}

The authors have no conflicts of interest to disclose.

\section{References}

1 Bulsara KR, Zomorodi A, Provenzale JM: Anatomic variant of the posterior cerebral artery. AJR Am J Roentgenol 2007;188:W395.

72 Moffat DB: The development of the posterior cerebral artery. J Ant 1961;95:485-494.

-3 Veras TW, Elhert GW: Variation of the posterior cerebral artery and its embryological explanation: a cadaveric study. Bol Asoc Med PR 2010;102:55-58.

-4 Krishnamurthy A, Nayak SR, Ganesh Kumar C, et al: Morphometry of posterior cerebral artery: embryological and clinical significance. Rom J Morphol Embryol 2008;49:43-45.

$>5$ Gailloud P, Clatterbuck RE, Fasel JHD, et al: Segmental agenesis of the internal carotid artery distal to the posterior communicating artery leading to the definition of a new embryologic segment. AJNR Am J Neuroradiol 2004;25:1189-1193.

6 Moyer DJ, Flamm ES: Anomalous arrangement of the origins of the anterior choroidal and posterior communicating arteries. Case report. J Neurosurg 1992;76:1017-1018.

-7 Pedroza A, Dujovny M, Artero JC, et al: Microanatomy of the posterior communicating artery. Neurosurgery 1987;20:228-235.

8 Berenstein A, Lasjaunias PL: Surgical Neuroangiography, ed 2. Berlin, Springer, 2004.

9 Shapiro M: Neuroangio.org. http://neuroangio.org/anatomy-and-variants/posterior-cerebral-artery (accessed April 30, 2015). 